\title{
Clock SyNCHRONIZATION USING TRUNCATED MEAN AND WHALE OPTIMIZATION FOR Clustered SENSOR NETWORKS
}

\author{
Karthik Soundarapandian ${ }^{1}$ and Ashok Kumar Ambrose ${ }^{2}$ \\ ${ }^{1}$ Research Scholar, Research and Development Centre, \\ Bharathiar University, Coimbatore, Tamilnadu, India \\ ${ }^{2}$ Assistant Professor, Department of Computer Science, \\ Alagappa Govt. Arts College, Karaikudi, Tamilnadu, India
}

\begin{abstract}
Clock synchronization is an important component in many distributed applications of wireless sensor networks (WSNs). The deprived method of clock offset and skew estimation causes inaccuracy, synchronization delay, and communication overhead in the protocols. Hence, this paper exploits two techniques of variation truncated mean (VTM) and whale optimization (WO) to enhance the synchronization metrics. Sensor nodes are grouped into several non-overlapped clusters. The cluster head collects the member nodes' local time and computes the synchronization time $S Z_{t}$ using the truncated mean method. Nodes with a high variation in the timings compared to a preset value are truncated. The head node broadcasts the $S Z_{t}$ in which the whale optimization is aiming at each node to reduce the synchronization error. The intra and inter-cluster synchronizations are accomplished through the multihop message exchange approach. The theoretical analysis is validated, and the simulation outcomes show that the performance metrics in the proposed work are better than the conventional methods by achieving minimum error value.
\end{abstract}

\section{KEYWORDS}

Clustering, Clock Synchronization, Truncated Mean, Whale Optimization, Clock Time Duration.

\section{INTRODUCTION}

The research focus on the sensor network domain has extensively increased for the use of prospective applications such as target tracking, industrial automation, area monitoring, military, and landslide detection. In these applications, nodes are necessitated to maintain a standard time to specify the timing of events [1]. Therefore, the clocks of the sensor nodes (SNs) are vital to synchronize which allows the entire network to collaborate and imparts reliable seamless communication between the nodes without delay. Time synchronization (TS) is a critical middleware package that requires consistent distribution sensing and control over large-scale sensor networks. TS may not be required in the centralized networks as there is no uncertainty. Over the past few years, several protocols have been proposed to address the synchronization problem like precision, computational complexity, node failure, and energy consumption [2].

The sensors' hardware clocks are imperfect and functioning at particular frequencies. Since nodes are deployed randomly at different positions, the temperature fluctuations may cause the clock to slow down or speed up. The frequency is the speed of clock ticks, and its alteration in the logical clocks minimizes the error [3]. Sensors maintain their clocks and sometimes obtain disparate 
timings due to: (i) clocks if started at different times (ii) quartz crystals in nodes might have been acted on different frequencies causing the clock values to diverge from each other, and (iii) the frequency of the clocks change over the time due to aging or ambient conditions. The nodes initiate the synchronization process periodically based on the clock readings, and the communication delay between two linked nodes is excluded in the delay-free case model [4,5]. The lifetime of synchronization is the amount of time that nodes maintain the clock readings consistently. Synchronization can be either global, where the timings are equalized in all the nodes, or local, that is equalization barely in a set of nodes. Though the clocks are synchronized by the message exchange method, packets are also to be synchronized before the transmission because sensed data often loses valuable context without accurate time information [6]. The synchronization shall be based on either a sender-receiver or receiver-receiver approach and accomplished via one-way message passing, multi-way broadcasting, and pair-wise synchronization. It is also possible with a timestamp technique that most of the TS protocols required [7-9].

Cluster-based sensor networks can perform accurate synchronization efficiently. The clustering technique has been extensively used to solve most of the synchronization issues. The clusters consist of cluster heads (CHs) and member nodes (MNs). The head nodes are initially synchronized to the base station (BS), and children nodes synchronize with their heads [10,11]. Such a method of synchronization eradicates message replication that enhances the convergence rate compared to the traditional topology. The synchronization protocols are categorized into reference and distributed-based models. The reference node comprises a pre-defined structure to attain network-wide synchronization whereas, nodes are distributed to scale and robust the entire network topology. In [12-14], reference nodes are acting as superior nodes that broadcast the synchronization message with timing information over the neighbours to cover the environment. A network that has minimal reference nodes reduces the energy dissipation drastically because it acquires a lesser number of communications. TS protocols have also been developed using consensus-based technique $[15,16]$. The concept of consensus is achieving trustworthiness by involving multiple distributed nodes containing the same time information. The determination of drift and skew errors, message delay, and oscillation of clock speed are neither designed nor discussed in the consensus-based synchronization. The robust TS [17] is developed based on maximum consensus considering the estimation and compensation of errors. As much as the offset and skew errors are minimized, the synchronization accuracy is increased.

The synchronization delay occurs by the accounting of transmission and propagation times and reduced at the sender and receiver nodes using the timestamp method that is efficiently devised based upon a linear clock model [18]. The synchronization process is incomplete if a parent node or reference node fails. Therefore, the dynamic method of selection may be an optimal solution to avoid failure. The recovery of data packets from the failure nodes is a challenging task in the synchronization phase. A protocol called self-recoverable TS [19] adopts a candidate node as a timer to check whether the synchronization is over. It selects a new root node by considering the energy level. The single-hop neighbouring pair-wise average transmission gives a quite difference in timings for the large-sized network. Due to this reason, multi-hop pair-wise synchronization with a short route is considered to transmit the sync messages. Also, the ondemand method [20] is adopted for a mutual coordination (a reactive mechanism) in a multi-hop communication. The propagation of a sync message may increase the message complexity either if the sensing area is enlarged or network size is increased [21].

In this paper, we develop a cluster-based VTM-WO protocol to solve the addressed problems of synchronization. The clock time is not modified, and there is no virtual clock in the proposed work. The sync packets are recorded by the clock time duration (CTD) model. The major contributions of our proposed work are the followings: 
- A standard procedure of the clustering process for energy-efficient transmission.

- Truncated mean method for clock parameters (offset and skew) estimation.

- Multi-hop based synchronization with fewer messages overhead.

- Whale optimization technique to reduce the synchronization error in both intra and inter-cluster communications.

- Evaluation of synchronization metrics to show the performance of the proposed work with conventional algorithms.

The rest of the paper is organized as follows: Section 2 presents the existing protocols for time synchronization and the problem statement. In section 3, the network and clock models are discussed under system setup. The proposed VTM-WO protocol for intra and inter-cluster synchronizations is described in section 4. Section 5 illustrates the simulation results of the performance parameters. At last, the conclusion is given in section 6 .

\section{Related Work ANd Problem Statement}

Time is an imperative factor in network operations. The sensor's clock is defined as a time calculator and a layer of the network architecture. Nodes' looping a time division multiple access (TDMA) protocol must agree for the time slots to avoid collision and overlapping hence, synchronization becomes essential for any successful event. This section discusses the recent works and problems related to synchronization in the distributed environment.

J. Wang, et al., [22] proposed the two-hop time synchronization (TTS) protocol for sensory networks where synchronization overhead is highly reduced. In TTS, the reference node records the timestamp, and the parent node synchronizes with the children nodes through two-way timing messages. The synchronization error is reduced by involving the minimum relay nodes. The problem with the protocol is much sensitive while changing the network size that led to more delays in the synchronization process. The clustering technique has been integrated into consensus-based time synchronization (CCTS) algorithm [23] to eradicate the message replications. The algorithm consists of two phases: (i) intra-cluster synchronization where the skew and offset parameters are updated by the average value of the virtual clocks (ii) inter-cluster synchronization in which head nodes' are assigned with weights based on the cluster sizes. The algorithm achieves faster convergence and better energy consumption. In CCTS, the synchronization error is not addressed due to the deprived method of clock estimation delay.

Clock skew is one of the important attributes for better accurate synchronization. F. Shi, et al., [24] presented maximum likelihood estimation (MLE) algorithm to reduce the skew convergence error based on the Gaussian distribution delay and multiple one-way broadcast models. The MLE performs accurate estimation of skew, quick convergence speed, and energy-efficient. Results for other synchronization parameters are not carried to analyze the overall performance of the algorithm. In [25], a field-programmable gate array (FPGA) method is introduced for timestamping pertained to reference broadcast synchronization (RBS). The authors also proposed a dynamic adjusting time synchronization (DATS) protocol for compensating the node clocks continuously through dynamic adjustments (coarse and fine). It does not work well for a largescale network during the synchronization phase. The prediction scheme over the skew or offset error in time synchronization does not modify or readjust in any of the parameters. H. Wang, et al., [26] employed a linear Gaussian delay model to estimate the skew convergence and aimed to adjust the clock immediately using a multi-hop approach. At every round of synchronization, a one-way timing message is distributed, and skew is estimated without considering the delay element. The message overhead and energy consumption are raised due to multiple intermediate nodes. The use of pulse-coupled synchronization to an acoustic event detection system for a 
International Journal of Computer Networks \& Communications (IJCNC) Vol.13, No.3, May 2021

wireless sensor network is presented by Nunez, F et al [27]. It aims at finding the source of acoustic events using the arrival time and also facilitates the accurate distributed localization even if there is a clock drift between the nodes. Each sensor creates a variable that increases at a specified rate by the frequency. The variable is initialized with the incoming pulses and broadcasts them to the neighbours. The authors use the consensus synchronization technique to attain both localization and synchronization through simple communication. In this algorithm, the mean absolute error (MAE) is not minimized in all the cases, and also the accurate localization is not compensated.

A single-hop communication in consensus-based algorithms performs defective results for both accuracy and convergence speed. N. Panigrahi and P.M. Khilar, [28] have proposed a consensusbased selective average time synchronization (SATS) algorithm for multi-hop networks. The algorithm effectively enhances the topological connectivity and restricts the end-to-end delay through a constraint-based dynamic programming approach. The multi-hop SATS is equipped with a rapid convergence however, message complexity is high. In [29], cluster-based synchronization is addressed called energy-based proportional integral and least common multiple (EPILCM) protocol. It compares the node's time with the reference clock and if there is any difference then, computes the propagation delay. The clock parameters are compensated using a proportional-integral controller, and the consensus-based synchronization is performed by the LCM method. The processing time of synchronization is relatively high in EPILCM.

G.S.S. Chalapathi, et al., [30] proposed an efficient and simple algorithm for time synchronization (E-SATS) to minimize the synchronization error and energy consumption in two different environments. In E-SATS, clusters are formulated by the head nodes, and the joining packets are broadcasted. If a node collects more than one acknowledgment from the heads such node becomes a gateway node. After the clustering process, the head node transmits a synchronization packet to its members containing a timestamp. All member nodes record the reception time and sending the acknowledgment packets after a pre-determined time. The clock skew and offset error rate are not methodologically computed thus, increase the synchronization complexity, and also precision is defected by the delay. In [31], rapid-flooding multi-broadcast time synchronization with real-time delay compensation is presented to reduce the packet delay in a high-density network. The protocol broadcast the redundant packets and estimate the accurate offset-skew using MLE. Also, a two-way exchange model is used to guarantee the delay estimate and the precision. The energy wastage is more due to message overhead.

We have addressed the problems of all the above existing synchronization protocols in this section. The lack of a solution to these problems and limitations motivated us to propose a proficient protocol for the clock synchronization component.

\section{SYSTEM SETUP}

The network is designed with the homogeneity of nodes where the base station is situated within the sensing environment. The clusters are non-overlapped by considering the overall energy consumption of the network. Both intra and inter-cluster synchronizations are executed based on the sender-receiver approach. The TDMA protocol is invoked to avoid the same time event occurrence in the multi-hop messages transmission. The section discusses the network depiction in a matrix structure, the method of clustering, and the CTD timestamp model for the cluster synchronization. 
International Journal of Computer Networks \& Communications (IJCNC) Vol.13, No.3, May 2021

\subsection{Network Model}

The sensing field is assumed as a random graph $G=(V, E)$, where $V$ symbolizes the group of nodes and $E$ represents the link matrix between the neighbouring nodes. The node is indexed by $n$ and it ranges from $v_{1}$ to $v_{n}$ that is expressed by $v_{n} \in V$. When a node $v_{m}$ is in transmission range with another node $v_{n}$ then, the link is created as $l_{m n}=1$ otherwise, $l_{m n}=0$, no connection exists. Each node is uniquely identified in the network. The communication between the linked nodes is symmetric, undirected, and static. The link $l_{m n}$ between nodes is represented in the $V \times V$ sparse matrix as:

$$
E=\left[\begin{array}{ccc}
l_{11} & \cdots & l_{1 n} \\
\vdots & \ddots & \vdots \\
l_{m 1} & \cdots & l_{m n}
\end{array}\right]
$$

\subsection{Clustering Phase}

The clustering technique provides optimal energy utilization, lesser communication overhead, and reduced crashing transmission. In this paper, non-overlapping clusters are formulated by following our previously proposed REEC non-overlap protocol [10]. The steps for clustering are:

Step 1 : Initially, BS identifies the centroid of a square sensing environment.

Step 2 : BS calculates the average distance between the centroid and the nodes using the euclidean metric.

Step $3: k$ number of clusters are determined using the energy communication model [32].

Step 4 : Mean points $M$ are initially selected for $k$ clusters.

Step 5 : Nodes are assigned to the closest $M$. This step is repeated until the static mean points are met.

Step 6 : After the cluster construction, BS determines the cluster heads $(\mathrm{CHs})$ for $k$ clusters considering the rating value $R_{v}$ of sensor nodes. The $R_{v}$ is computed based upon the three parameters such as available energy (AE), node's degree (ND), and node's distance to BS (d_BS).

Step 7 : A node that has higher $R_{v}$ would be elected as a $\mathrm{CH}$ in the respective cluster. The $\mathrm{CHs}$ are rotated to balance the energy and enhance the lifetime.

Such a method of clustering has proved as a better energy-efficient protocol in [10], and also enhances the network lifetime, minimized delay, and maximized delivery ratio. Figure 1 shows the clusters where the sensing area is $250 \times 250$ in meters, $k=7$ and BS (Sink node) is positioned at $240 \times 240(\mathrm{~m})$. 


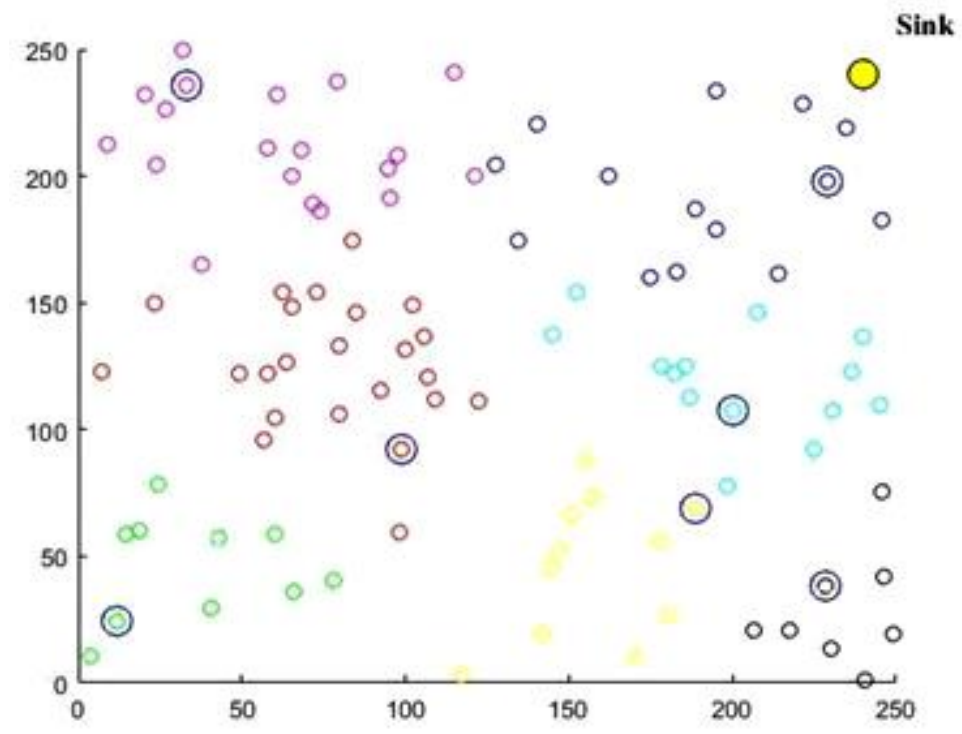

Figure 1. Clusters with cluster heads

\subsection{Clock Model}

Typically, a node's time $C(t)$ is composed of a local hardware-based crystal oscillator and a counter [1]. Such a physical clock is used for tracking the current time of nodes in the sensor network. At the initial stage, the time is set uniformly in all the nodes $V$ however; there will be a drift in the clocks after a certain time due to signal variations. The local clock of actual initial time $t$ in each sensor is mathematically given as:

$$
C(t)=\propto \int_{0}^{t} \omega(t) d t+C\left(t_{0}\right)
$$

where $\propto$ is a coefficient of proportionality, $\omega(t)$ denotes the oscillator's angular frequency and $C\left(t_{0}\right)$ represents the initial time of a sensor's clock. In this paper, the communication is recorded by a clock time duration (CTD) model as a timestamp shown in figure 2 . The timestamp is an important element to achieve synchronization, and to maintain the clocks. The CTD of a member node $n$ and a head node $h$ is formulated by compiling both clocks offset and skew as:

$$
C_{n}(t)=C_{h}(t)=\lambda_{n}+\gamma_{n} t
$$

where $\lambda_{n}$ represents the clock offset that is a variation from the real time, and $\gamma_{n}$ is the clock skew that defines the clock frequency signal arrives at different components. Assume that there are two nodes namely $V_{1}$, and $V_{2}$ with CTDs represented by $C_{1}(t)$ and $C_{2}(t)$ comprising equivalent signal frequencies that is, expressed as:

$$
\gamma_{1} C_{1}(0)=\gamma_{2} C_{2}(0)
$$

The clocks offset and skew between two nodes $V_{1}$ and $V_{2}$ are computed using the following Eq. (4) and Eq. (5):

$$
\begin{gathered}
\lambda_{n}=\left|C_{1}(t)-C_{2}(t)\right|=\left|\lambda_{1}-\lambda_{2}\right| \\
\gamma_{n}=\left|\gamma_{1} C_{1}(t)-\gamma_{2} C_{2}(t)\right|
\end{gathered}
$$


International Journal of Computer Networks \& Communications (IJCNC) Vol.13, No.3, May 2021

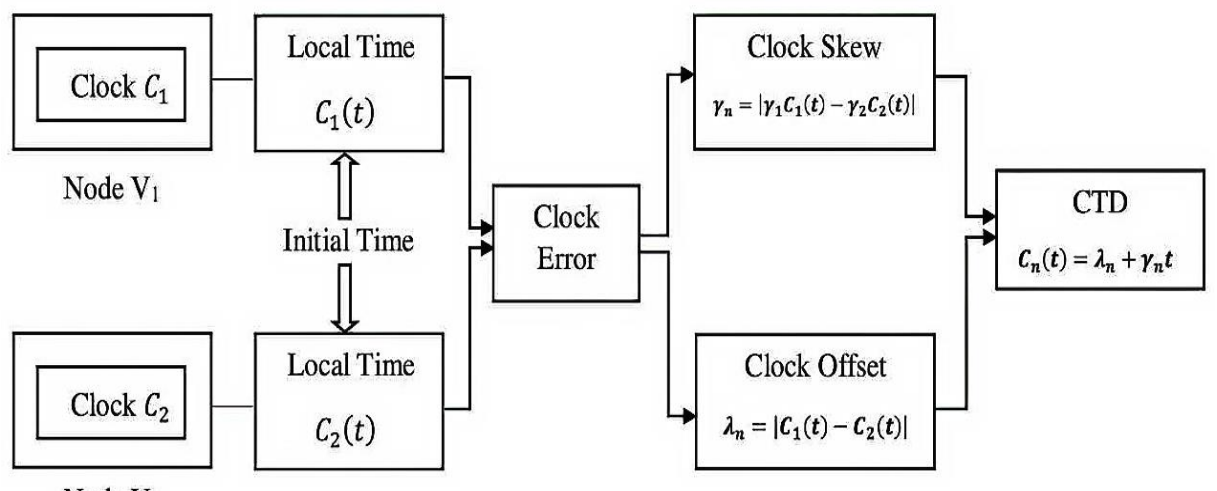

Figure 2. The CTD of clock offset and skew

\section{Proposed VTM-WO Protocol for Clock Synchronization}

In this section, we first discuss the variation truncated mean to determine the synchronization time and then present the whale optimization algorithm to minimize the sync errors in both intra and inter-cluster synchronization phases. Figure 3 illustrates the overall process of the VTM-WO protocol.
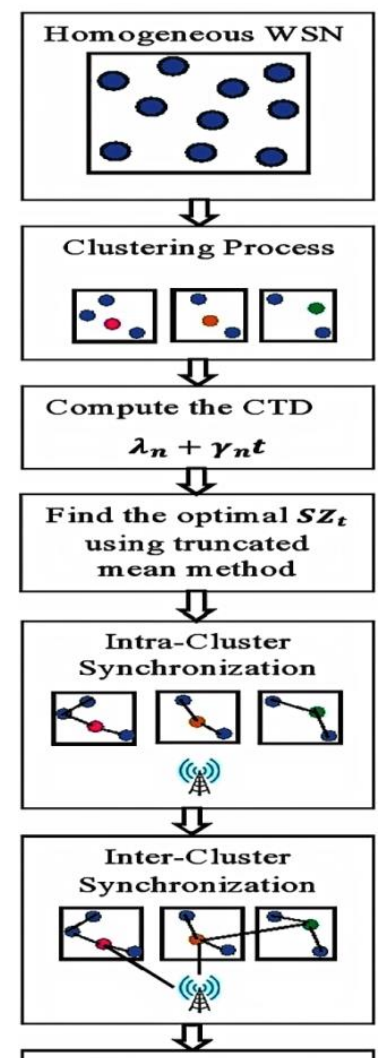

$\boldsymbol{S} \boldsymbol{Z}_{\boldsymbol{e}}$ minimization

using whale optimization

Figure 3. Flow chart for VTM-WO protocol 
International Journal of Computer Networks \& Communications (IJCNC) Vol.13, No.3, May 2021

\subsection{Truncated Mean Approach for Synchronization Time}

The maximum time variation factor among the nodes' clock implies an inaccuracy in determining the synchronization time $S Z_{t}$. Hence, we use the truncated function with the mean $\mu$ to synchronize the sensor node's CTD. In clock synchronization, the mean is an average of a collection of clock times. The truncated mean (TM) is similar to a mean however; it involves the computation of mean after discarding the probability outliers. In the proposed VTM-WO method, TM is to be considered as an estimator for both clock offset and skew. For instance, consider a homogeneous network that consists of a cluster of five nodes $V_{1}, V_{2}, V_{3}, V_{4}$, and $V_{5}$ with their local time as $9.04,9.08,11.45,9.07$, and 7.22 , respectively. The two values are varying much compared to the other three values wherein the mean $\mu$ is computed and resulting in 9.172. Therefore, applying the arithmetic mean will not be feasible in this synchronization computation because it led to high variation and mortify the synchronization accuracy. To eliminate the time offset, we invoke the truncated mean method in which the highest and the lowest time values are discarded. So, the time values of $V_{1}, V_{2}$, and $V_{4}$ are considered for the computation of $S Z_{t}$ whereas $V_{3}$ and $V_{5}$ values are removed. The result obtains the optimal time value 9.063 as the $S Z_{t}$. It is proved that the outliers of time variation are minimized and the accuracy of sync time is maximized by employing the truncated mean. The synchronization factor $\infty$ is identified as $0.023,0.017$, and 0.007 by obtaining the variation between CTD and $S Z_{t}$. Based on these variation times, the nodes' clocks are adjusted to a common time using the whale optimization algorithm discussed in section 4.4. The truncated mean function is formulated as:

$$
\operatorname{Trunc}_{\mu}\left(S Z_{t}\right)=\frac{\sum C_{V}(t)-\left(L C_{n}(t)+H C_{n}(t)\right)}{V-2}
$$

where $C_{V}(t)$ is the clock time of all sensor nodes, $L C_{n}(t)$ and $H C_{n}(t)$ represents the lowest and highest time of the nodes. The execution of the VTM approach is detailed in the intra and intercluster synchronization sections.

\subsection{Multi-hop Intra-Cluster Synchronization Phase}

The intra-cluster synchronization is a clock synchronization in which a head node initiates the process with member nodes in a cluster. Such a node allocates the disparate time slots as shown in figure 4 to transmit and receive the synchronization messages following TDMA format. The head node transmits a synchronization message to its member nodes through the multi-hop approach when both nodes are not in the transmission range. After receiving that message, the MNs compute the CTD timestamp and transmit it to the corresponding cluster head within the stipulated time. Hence, there is no chance for the occurrence of collision in the network. The head node receives the CTD sequentially and computes the variation by combining the skew parameter $\gamma_{n}$ and $C_{n}(t)$. 


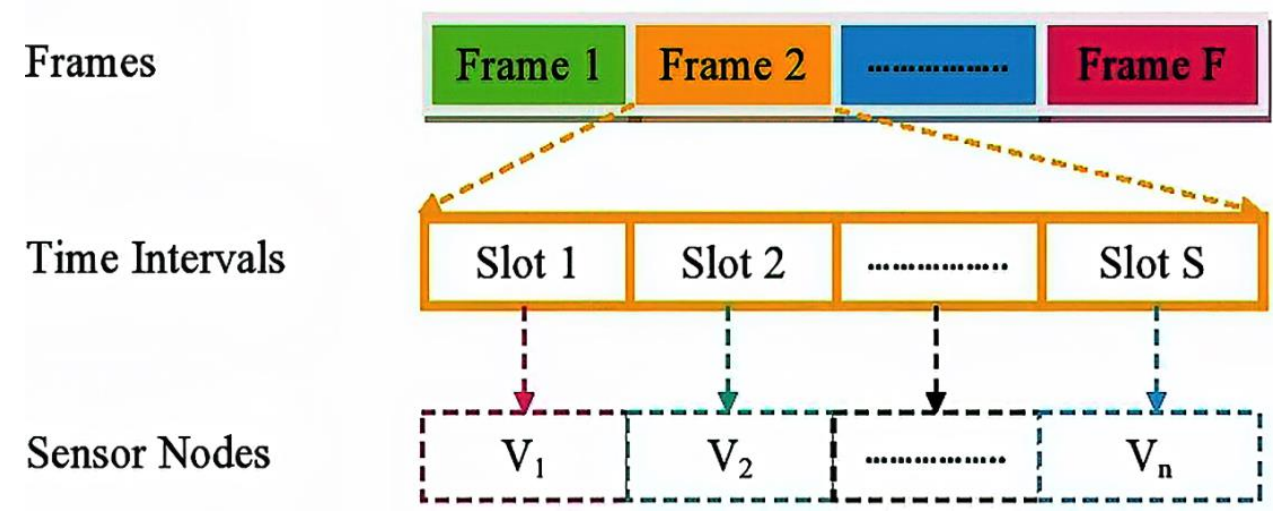

Figure 4. TDMA for synchronization message exchanges

The truncated mean is employed to compute a common time that time value is ascertained as the optimum $S Z_{t}$ in the intra-cluster synchronization. After executing the VTM, the sync time is updated within the cluster and the clock offset $\lambda_{n}$ is eradicated. The time variation $t_{v}$ has a predefined value in which any member node obtains a greater time value to synchronization factor $\infty_{n}$ that is, the time difference between CTD and $S Z_{t}$ then, whale optimization algorithm discussed in section 4.4 is executed to minimize the error. The distance of the $\mathrm{CH}$ to its member nodes is very proximity therefore, the propagation time and the message transmissions are highly minimized in the intra-cluster synchronization. The operational principle of intra-cluster synchronization is given in the below pseudo code.

\section{Pseudo Code 1: Intra-Cluster Clock Synchronization}

1. Start procedure

2. Consider a set of nodes $S_{n}$ in a cluster containing a head node $h$ and $n=1,2,3, \ldots, S$.

3. for each $n$ do

4. Broadcast the sync_req from $h$ containing $t_{1}$ timestamp following the multihop.

5. Node $n$ records the $t_{1}$ and acknowledges sync_ack message to $h$.

6. $\quad$ Calculate the CTD $\left(t_{1}, t_{2}, t_{3, \ldots}, t_{S}\right)$ using $C_{n}(t)$.

7. $\quad$ if $\left(C_{n}(t)<t_{v}\right)$ then $/ / t_{v}$ is the time variation in seconds

8. $\quad$ Calculate $\mu^{\prime}=\sum_{n=1}^{S} \frac{C_{n}(t)}{S}$

9. $\quad$ else

10. $\quad$ Execute the truncated mean function using Eq. (6).

11. Head node $h$ broadcast the sync_pack to its member nodes.

12. end if

13. Evaluate the synchronization factor $\infty_{n}=C_{n}(t)-S Z_{t}{ }^{\prime}$

14. $\quad$ if $\left(\infty_{n}<t_{v}\right)$ then

15. $\quad \infty_{n}=\infty_{n}$ // Update the sync time

$16 . \quad$ else

17. Execute the whale optimization algorithm.

18. $\quad \infty_{n}=\infty_{n}-t_{v}$

$19 . \quad$ end if

20. end for

21. End procedure 
International Journal of Computer Networks \& Communications (IJCNC) Vol.13, No.3, May 2021

\subsection{Multi-hop Inter-Cluster Synchronization Phase}

The cluster heads that are closer to the base station can transmit the synchronization messages in a single-hop however the distant nodes follow the multi-hop approach. All head nodes in the inter-cluster do not receive the sync_message concurrently rather receive at different times and records the timestamp. In intra-cluster, member nodes are synchronized in disparate $S Z_{t}$ with their corresponding $\mathrm{CHs}$ but head nodes are synchronized in a uniform time with the base station. Like the intra-cluster synchronization process, the VTM method is applied to compute the synchronization time based on the CTD, and whale optimization is employed to reduce the synchronization error at each $\mathrm{CH}$. The step-by-step procedure followed for inter-cluster synchronization is delineated in pseudo code 2 .

\section{Pseudo Code 2: Inter-Cluster Clock Synchronization}

1. Start procedure

2. Consider a set of $k_{h}$ head nodes in the network where $h=1,2,3, \ldots ., k$

3. for each $h d o$

4. $\quad$ BS broadcast the sync_req containing $t_{1}{ }^{\prime}$ timestamp following the multi-hop.

5. $\quad k$ nodes transmit the sync_ack in different timings.

6. $\quad$ Compute the CTD $\left(t_{1}{ }^{\prime}, t_{2}{ }^{\prime}, t_{3}{ }^{\prime}, \ldots . .,{ }_{k}{ }^{\prime}\right)$ using $C_{h}(t)$.

7. $\quad$ if $\left(C_{h}(t)<t_{v}\right)$ then

8. $\quad$ Calculate $\mu^{\prime \prime}=\sum_{h=1}^{k} \frac{C_{h}(t)}{k}$

9. $\quad$ else

10. $\quad$ Execute the truncated mean function using Eq. (6).

11. $\quad$ BS broadcast the sync_pack to $k$ cluster heads.

12. end if

13. Evaluate the synchronization factor $\infty_{h}=C_{h}(t)-S Z_{t}{ }^{\prime \prime}$

14. $\quad$ if $\left(\infty_{h}<t_{v}\right)$ then

15.

16. else

17. Execute the whale optimization algorithm.

18. $\quad \infty_{h}=\infty_{h}-t_{v}$

19. $\quad$ end if

20. end for

21. End procedure

\subsection{Synchronization Error Minimization using Whale Optimization}

Optimization techniques are proved in resolving different issues of network operations. As synchronization error $S Z_{e}$ is constantly challenging in the existing synchronization protocols, a recently presented whale optimization algorithm (WOA) is integrated to minimize the errors. The WOA [33] describes the hunting behaviour of humpback whales for the prey in which three phases are segmented such as encircling, bubble-net feeding, and searching. In the proposed VTM-WO, the prey is a sync time, humpback whales are sync errors, and the fitness function $F$ is to reduce the errors between actual and estimated time variation factors. The CTD, $S Z_{t}$, and $\infty$ are assumed as the input to a population of WOA. The fitness value is computed to minimize the occurring errors while updating the sync time that is given as:

$$
F_{\min }=\frac{1}{V} \sum_{n=1}^{V}\left|A_{n}-E_{n}\right|
$$


where $V$ indicates the total number of nodes, $A$ and $E$ represents the actual and estimated time of the message transmission and $\left|A_{n}-E_{n}\right|$ is referred to $S Z_{e}$.

Phase 1: Encircling the prey - The whales commence the hunting process in which the present location is considered as the best solution or close to the optimum region. After the best position is identified then, the whales are moving towards it. This performance is applied in VTM-WO as: The nodes' current time is a source to compute a sync time and time variations are equalized when member nodes update the new sync time $S Z_{t}$ that is considered as the best time in the network. Such a process is expressed as:

$$
\begin{gathered}
\infty=\left|X \times t^{b}(i)-t(i)\right| \\
t(i+1)=t^{b}(i)-Y \times \infty
\end{gathered}
$$

where $i$ represents the iteration, $t^{b}$ defines the best time that requires to be updated in the network, $t$ indicates the current time, $X$ and $Y$ denotes the coefficient vectors that are computed as:

$$
\begin{gathered}
X=2 x \times y-x \\
Y=2 \times y
\end{gathered}
$$

where $x$ is sequentially reduced from 2 to 0 over the periods and $y$ represents a random number in the range $[0,1]$.

Phase 2: Bubble-net attacking behaviour - This is referred to as the exploitation phase that comprises two imperative mechanisms.

- Shrinking encircling prey mechanism: This approach shrinks the sync errors by reducing the value $x$ given in the Eq. (12). Subsequently, $Y$ also is reduced in the range $[-x, x]$ from 2 to 0 . The new sync time $\left(t^{b}\right.$ and CTD) is defined when the random values for $Y$ in $[-1,1]$.

- Updating spiral position mechanism: This approach computes the time variation between $t$ (whale position), and $t^{b}$ (prey position). The spiral equation between these two is derived as:

$$
t(i+1)=\infty^{\prime} \times e^{p q} \times \cos (2 \pi q)+t^{b}(i)
$$

where $\infty^{\prime}=\left|t^{b}(i)-t(i)\right|, p$ is a constant value for the logarithmic spiral and $q$ is a random number in the interval $[-1,1]$. We assume that there is a $50 \%$ possibility to select any one of the mechanisms to update the sync time while performing optimization and is formulated as given below:

$$
t(i+1)= \begin{cases}t^{b}(i)-Y \times \infty & \text { if } Z<0.5 \\ \infty^{\prime} \times e^{p q} \times \cos (2 \pi q)+t^{b}(i) & \text { if } Z \geq 0.5\end{cases}
$$

where $Z$ is in [0,1] means, $t^{b}$ search for CTD to quantify the synchronization factor $\infty_{n}$. 
Phase 3: Search for prey - It is also known as the exploration phase. In the network, nodes are searching for the best $t^{b}$ randomly because the optimal sync time is not known. This phase exploits the variation of $Y$ in interval [-1,1] to update the best time in the randomly selected nodes. These two activities are aiming to minimize the errors that are expressed as:

$$
\begin{gathered}
\infty=\left|X \times t_{\text {rand }}-t\right| \\
t(i+1)=t_{\text {rand }}-Y \times \infty
\end{gathered}
$$

The current time of the nodes are randomly chosen from the network population and is represented as $t_{\text {rand }}$. The fitness function of the WOA algorithm for minimizing synchronization errors is robust and efficient. Hence, this optimization technique can provide a faster convergence speed by the low time-variation error factor. The pseudo-code for WOA-based synchronization error minimization is given as follows:

\section{Pseudo Code 3: WOA-Based Synchronization Error Minimization}

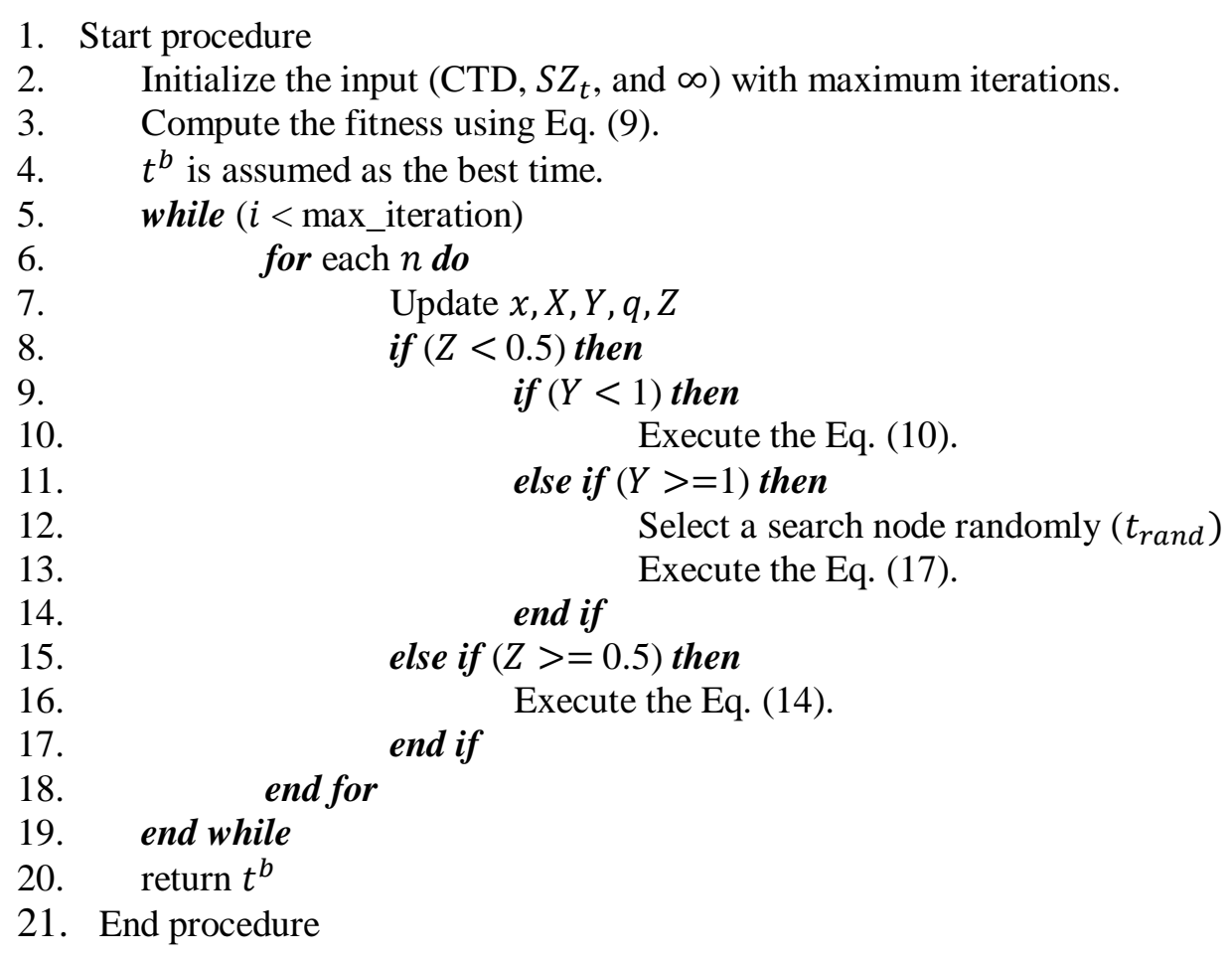

\subsection{Average Synchronization Error Analysis}

Typically, a message transmission through multiple intermediate nodes would persuade the delay error due to the propagation time component in particular. The timestamp CTD is a vital parameter to compute the $S Z_{e}$. The arrival time of a sync_pack and the expected time of delivery may cause the time variation during the synchronization in the proposed VTM-WO protocol. Therefore, it is important to analyse the time utilized to calculate and update the sync time among the nodes. Let assume a cluster consists of $S$ nodes for intra-cluster and $k$ cluster heads for intercluster that are required to sync with $S Z_{t}{ }^{\prime}$ and $S Z_{t}{ }^{\prime \prime}$ respectively, in the network. The average of 
International Journal of Computer Networks \& Communications (IJCNC) Vol.13, No.3, May 2021

the relative clock for intra-cluster $S Z_{e}{ }^{\prime}$ and inter-cluster $S Z_{e}{ }^{\prime \prime}$ synchronization errors are as follows:

$$
\begin{aligned}
S Z_{e}{ }^{\prime} & =\int_{n=1}^{S} \frac{\left[C_{n}(t)-C(t)\right]}{S} \\
S Z_{e}{ }^{\prime \prime} & =\int_{h=1}^{k} \frac{\left[C_{h}(t)-C(t)\right]}{k}
\end{aligned}
$$

where $C_{n}(t), C_{h}(t)$ are defined in Eq. (2), and $C(t)$ is given in Eq. (1). When a head node $h$ and BS initiates the sync process, the multi-hop VTM-WO protocol repeats a sync_message transmission till the process is completed.

$$
\begin{gathered}
S Z_{e}{ }^{\prime}=\int_{n=1}^{S} \frac{\left[\infty_{n}\left(\left(\lambda_{n}+\gamma_{n} t\right)-S Z_{t^{\prime}}\right)\right]_{i}^{j}}{S} \\
S Z_{e}{ }^{\prime \prime}=\int_{h=1}^{k} \frac{\left[\infty_{h}\left(\left(\lambda_{h}+\gamma_{h} t\right)-S Z_{t^{\prime \prime}}\right)\right]_{i}^{j}}{k}
\end{gathered}
$$

The synchronization factor $\infty$ for both intra and inter is delineated in section 4.4., the sync times $S Z_{t}{ }^{\prime}, S Z_{t}{ }^{\prime \prime}$ are derived from using Eq. (6) at iteration $i=1$ to $j$, maximum iteration. The time variation $t_{v}$ is a constant predefined value, magnitude in $\infty$ and $S Z_{t}$ when the iteration is ended, that is given as:

$$
\begin{gathered}
S Z_{e}{ }^{\prime}=\int_{n=1}^{S} \frac{\left[\frac{\infty_{n}}{t_{v}}\left(\left(\lambda_{n}+\gamma_{n} t\right)-\frac{S Z_{t^{\prime}}}{t_{v}}\right)\right]}{S} \\
S Z_{e}{ }^{\prime \prime}=\int_{h=1}^{k} \frac{\left[\frac{\infty_{h}}{t_{v}}\left(\left(\lambda_{h}+\gamma_{h} t\right)-\frac{S Z_{t^{\prime \prime}}}{t_{v}}\right)\right]}{k}
\end{gathered}
$$

The $\lambda_{n}$, and $\lambda_{h}$ are eradicated because their values become 0 once the sync time is computed hence, it is defined as $S Z_{t}=\gamma t \times \infty$. This activity is applied in Eq. (22) and Eq. (23) then, we obtain

$$
\begin{gathered}
S Z_{e}{ }^{\prime}=\int_{n=1}^{S} \frac{\left[\frac{\infty_{n}}{t_{v}}\left(\left(\gamma_{n} t\right)-\frac{\gamma_{n} t \times \infty_{n}}{t_{v}}\right)\right]}{S} \\
S Z_{e}{ }^{\prime \prime}=\int_{h=1}^{k} \frac{\left[\frac{\infty_{h}}{t_{v}}\left(\left(\gamma_{h} t\right)-\frac{\gamma_{h} t \times \infty_{h}}{t_{v}}\right)\right]}{k}
\end{gathered}
$$

The sync factor value is rounded off to a certain fractional point and is estimated by:

$$
\begin{aligned}
& S Z_{e}{ }^{\prime}=\int_{n=1}^{S} \frac{\gamma_{n} t\left[\frac{\infty_{n}}{t_{v}}-\frac{\infty_{n}}{t_{v}}\right]}{S} \\
& S Z_{e}{ }^{\prime \prime}=\int_{h=1}^{k} \frac{\gamma_{h} t\left[\frac{\infty_{h}}{t_{v}}-\frac{\infty_{h}}{t_{v}}\right]}{k}
\end{aligned}
$$

Hence, the average synchronization errors for intra and inter-cluster synchronizations are $S Z_{e}{ }^{\prime} \approx$ 0 and $S Z_{e}{ }^{\prime \prime} \approx 0$. 


\section{RESUltS AND OBSERVATIONS}

In this section, the proposed VTM-WO has experimented with the standard existing TS protocols such as CCTS [23], TTS [22], and EPILCM [29] using MATLAB 2016a. The nodes' random deployment in WSN does not guarantee the complete coverage of the network, and VTM-WO consists of un-even sized clusters. The performance metrics considered for comparison are clock offset, clock skew, synchronization error, synchronization delay, communication overhead, energy consumption, and overall processing time. The results of these metrics are illustrated with the diagrams in the following subsections. The simulation parameters are depicted in Table 1.

Table 1. Simulation parameters

\begin{tabular}{|l|l|}
\hline Parameter & Values \\
\hline Sensing area & $250 \mathrm{~m} \times 250 \mathrm{~m}$ \\
\hline Number of nodes & $50,100,150,200,250$ \\
\hline Sink position (BS) & $240 \mathrm{~m} \times 240 \mathrm{~m}$ \\
\hline Transmission range & $15 \mathrm{~m}$ \\
\hline Deployment of nodes & Random \\
\hline MAC protocol & TDMA \\
\hline
\end{tabular}

\subsection{Clock Offset and Skew Convergence}

The less count of difference among the node's local clocks enhances the synchronization precision. VTM-WO is a master-slave synchronization architecture in which every head node calculates the offset and skew convergences of member nodes using the CTD method. The network's convergence rate increases only if clock offset and skew errors are reduced.

Figure 5 demonstrates the results of the clock offset and skews in nanoseconds (ns) for the 250 nodes' density. The time over the offset and skew are estimated effectively by utilizing truncated mean square error. As shown in figure 5.a., the clock offset values are 0.57(ns), 0.68(ns), 0.79(ns), and 0.90(ns) for VTM-WO, CCTS, TTS, and EPILCM, respectively. The variation in time between the nodes' clock frequencies is shown in figure 5.b. The skew convergence for the proposed algorithm is $1.07(\mathrm{~ns})$, and other conventional protocols are 1.50(ns), 2.59(ns), and 3.68(ns). Hence, the convergence is quicker in VTM-WO.

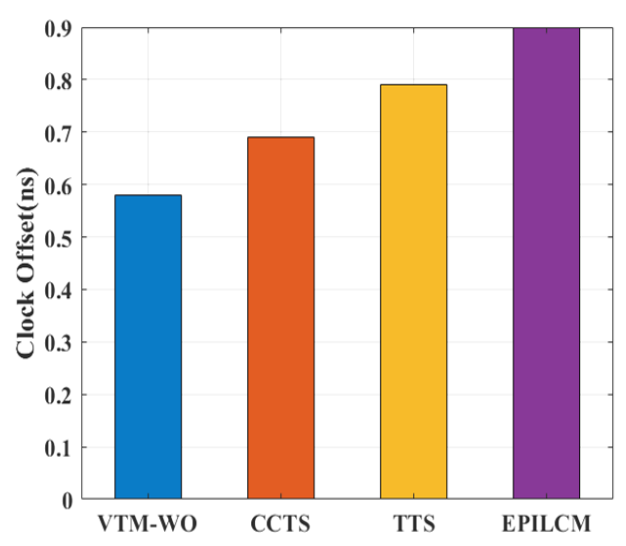

5.a. Offset

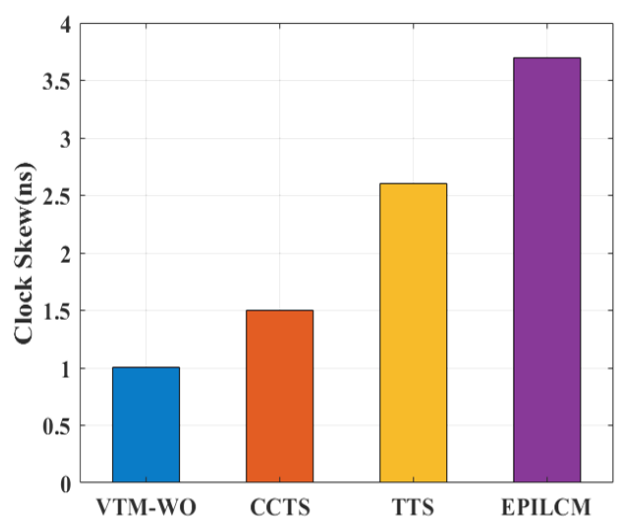

5.b. Skew

Figure 5. Clock convergence 


\subsection{Synchronization Error}

The time variation between the CTD and ST is the synchronization error measured in seconds (s). It is quantified for the network size of 250 nodes. The errors are added gradually when the hops are increased due to the distance factor shown in Figure 6. However, the error rates against the hops are not much as the network size is constant and the nodes are immobility. The errors are relatively high in all the conventional protocols compared to the proposed method because sync messages were crashed and necessitated to resent the same message.

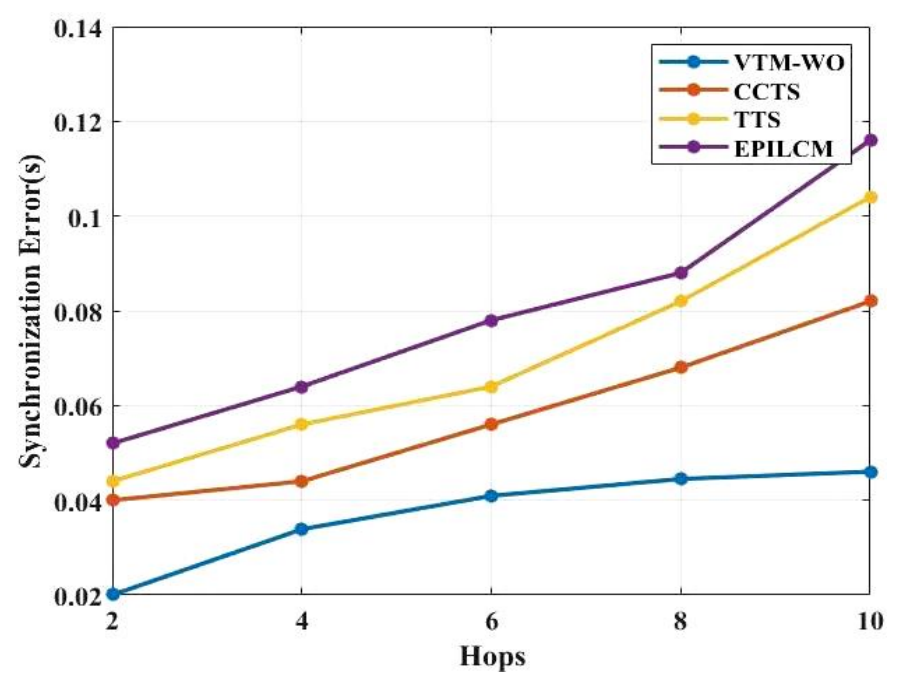

Figure 6. Synchronization error by varying multiple hops

From Table 2, the average synchronization error is computed as 0.0374(s), 0.0565(s), 0.0693(s), and 0.0787(s) for VTM-WO, CCTS, TTS, and EPILCM, respectively. The load balancing among the nodes and the distance proximity of neighbouring nodes are the two notable reasons for lower synchronization error in the proposed scheme.

Table 2. Synchronization error (s)

\begin{tabular}{|l|l|l|l|l|}
\hline Hops & VTM-WO & CCTS & TTS & EPILCM \\
\hline 2 & 0.0201 & 0.0398 & 0.0424 & 0.0531 \\
\hline 4 & 0.0332 & 0.0418 & 0.0562 & 0.0620 \\
\hline 6 & 0.0386 & 0.0549 & 0.0618 & 0.0783 \\
\hline 8 & 0.0443 & 0.0646 & 0.0815 & 0.0836 \\
\hline 10 & 0.0512 & 0.0814 & 0.1047 & 0.1166 \\
\hline
\end{tabular}

\subsection{Synchronization Delay}

The contrary proportionality of estimated synchronization time is the synchronization delay. In this paper, the delay metric is measured in microseconds (ms) and experimented with by varying from 50 to 250 nodes. Figure 7 shows the synchronization delay, and the parameter is gradually heaving when the network density is increased in the sensing area. 


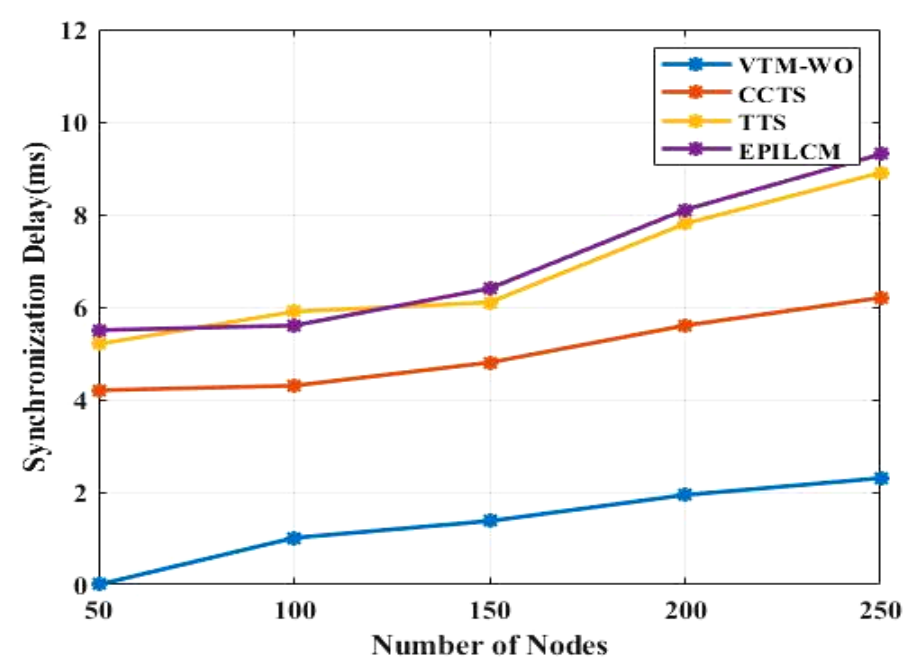

Figure 7. Synchronization delay for proposed and existing approach

The head node's localization and its optimal multi-hop communication with the member nodes in VTM-WO reduces the synchronization delay by $72.03 \%, 79.29 \%$, and $79.88 \%$ comparing to CCTS, TTS, and EPILCM, respectively. Since the delay is highly less referred to in Table 3, the convergence speed is faster in VTM-WO.

Table 3. Synchronization delay (ms)

\begin{tabular}{|l|l|l|l|l|}
\hline Network Size & VTM-WO & CCTS & TTS & EPILCM \\
\hline 50 & 0.0004 & 4.2 & 5.2 & 5.5 \\
\hline 100 & 1.03 & 4.3 & 5.9 & 5.6 \\
\hline 150 & 1.59 & 4.8 & 6.1 & 6.4 \\
\hline 200 & 2.1 & 5.6 & 7.8 & 8.1 \\
\hline 250 & 2.3 & 6.2 & 8.9 & 9.3 \\
\hline
\end{tabular}

\subsection{Communication Overhead}

The number of synchronization message exchanges within and between the clusters per round is communication overhead in VTM-WO. It is computed with $k$ clusters and $V$ nodes. Figure 8 shows the communication overhead for different network sizes in which messages are hoisted for increasing sizes. Due to the proximity between heads and member nodes in both intra and interclusters, the proposed method acquires a lesser number of messages for the synchronization shown in Table 4. 


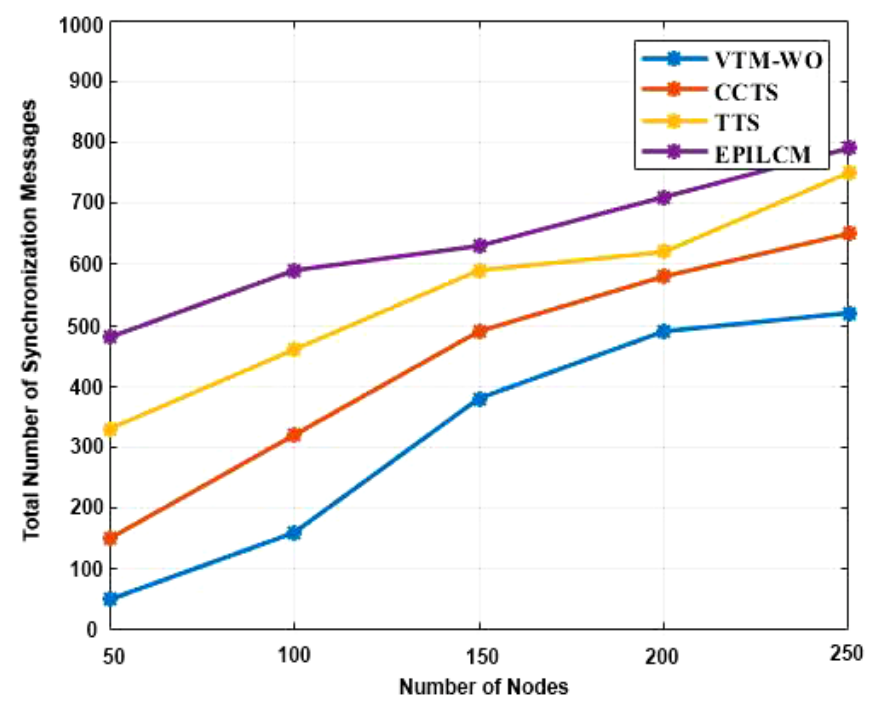

Figure 8. Synchronization messages for different size

All network size together, VTM-WO diminishes the synchronization message overhead by $26.82 \%, 41.75 \%$, and $49.67 \%$ as compared to CCTS, TTS, and EPILCM. This phenomenon result is reflected in energy dissipation and overall processing time.

Table 4. Communication overhead (Number of messages exchanged)

\begin{tabular}{|l|l|l|l|l|}
\hline Network Size & VTM-WO & CCTS & TTS & EPILCM \\
\hline 50 & 57 & 153 & 332 & 481 \\
\hline 100 & 158 & 316 & 466 & 589 \\
\hline 150 & 382 & 497 & 591 & 622 \\
\hline 200 & 491 & 582 & 616 & 709 \\
\hline 250 & 519 & 648 & 754 & 792 \\
\hline
\end{tabular}

\subsection{Energy Consumption}

The energy depletion is computed by the mathematical derivations discussed in [10]. Figure 9 obtains the energy usage for different sizes during synchronization in which the power dissipation is increased exponentially between 200 and 250 nodes due to communication overhead. It is observed that when the network size is enlarged then the involvement of transmitters and receivers is also increased which led to more energy dissipation. The proposed method dissipates less energy due to the minimized synchronization of message exchanges at all the network sizes. For example, at network size 150, VTM-WO consumed 0.2777(J) whereas 0.7359(J), 0.7847(J), and $0.9374(\mathrm{~J})$ by CCTS, TTS, and EPILCM, respectively. 
International Journal of Computer Networks \& Communications (IJCNC) Vol.13, No.3, May 2021

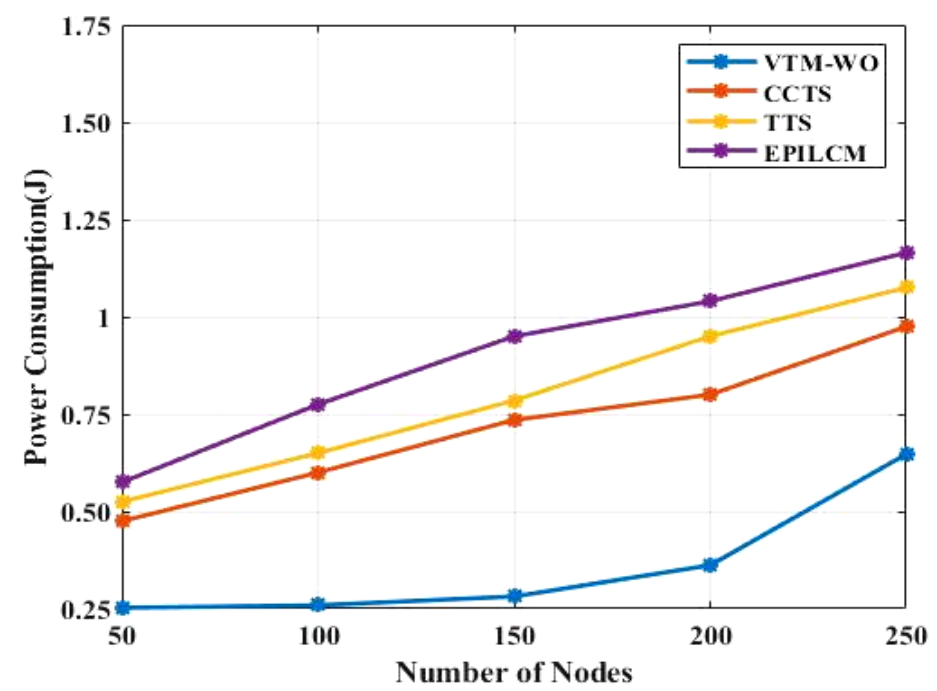

Figure 9 . Energy usage by varying nodes

\subsection{Overall Processing Time}

The overall processing time is the time engaged to complete the entire clock synchronization process. It is measured in seconds for the network size of 50 nodes. Figure 10 shows the processing time, and this parameter alone can determine the overall performance of the algorithms. When the proposed VTM-WO method is executed, it acquires $0.038(\mathrm{~s})$, and other algorithms obtain 0.081(s) for CCTS, 0.093(s) for TTS, and 0.102(s) for EPILCM, respectively. Due to less consumption of processing time, VTM-WO is efficient than other conventional methods.

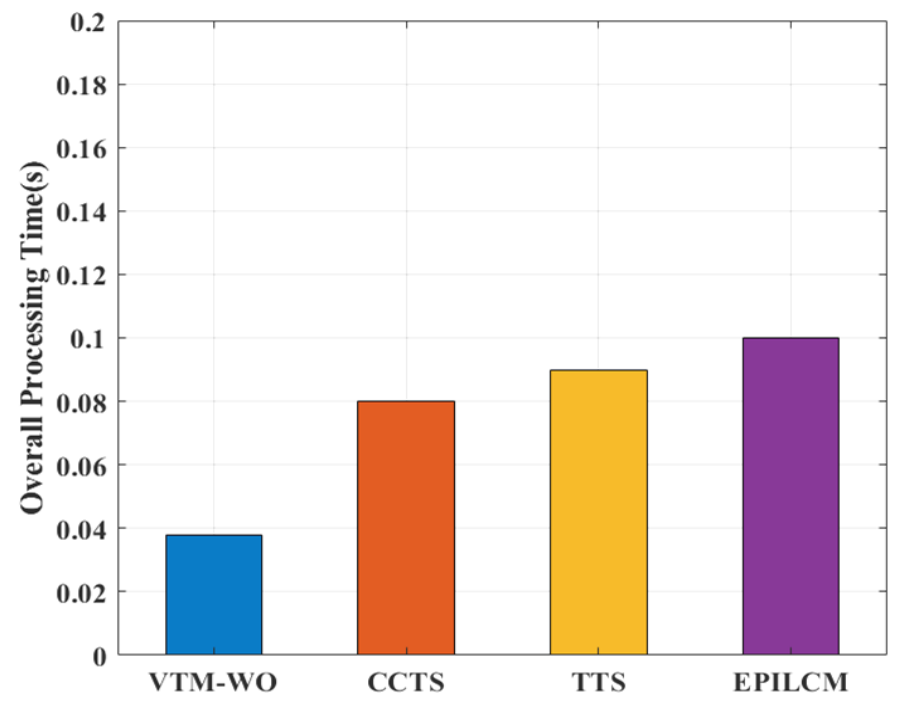

Figure 10. Processing speed for 50 nodes 
International Journal of Computer Networks \& Communications (IJCNC) Vol.13, No.3, May 2021

\section{CONCLUSIONS AND FUTURE WORK}

The proposed VTM-WO achieves the sender-to-receiver synchronization among the nodes in the clusters. The protocol incorporates the REEC non-overlap clustering to actualize the entire homogeneous network as energy-efficient. The CTD variations in both intra-cluster and intercluster synchronizations are compensated with a standard time using the truncated mean function. Also, the synchronization factor between $C_{n}(t)$ and $S Z_{t}$ is carried out in less error by the whale optimization's fitness function. The proposed work has been evaluated and compared with CCTS, TTS, and EPILCM. Simulation results show that the clock offset and skew errors are minimized in VTM-WO than the conventional protocols. Further, multi-hop message exchanges are attained at a lesser hop count that reduces the message transmission overhead. The experimental outcomes demonstrate that the proposed protocol synchronizes the local clocks with the least amount of delay and power consumption in the network. The overall performance of VTM-WO is highly improved where can implement in many applications of WSNs. However, the proposed scheme has some limitations such as the synchronization delay increases abruptly due to multiple message exchanges when the network size is augmented, and the nodes' energy is decreased rapidly if the transmission range is high. The future work will be focused on dynamic cluster topology with the resynchronization loop for the heterogeneous network considering the delay parameter.

\section{CONFLICTS OF INTEREST}

The authors declare no conflict of interest.

\section{REFERENCES}

[1] B. Sundararaman et al., (2005) "Clock synchronization for wireless sensor networks: a survey", $A d$ Hoc Networks, Vol. 3, No. 3, pp. 281-323.

[2] M. Xu, et al., (2016) "Energy-efficient time synchronization in wireless sensor networks via temperature-aware compensation", ACM Transactions on Sensor Networks, Vol. 12, No. 2, pp. 1-29.

[3] K. S. Yildirim, (2016) "Gradient descent algorithm inspired adaptive time synchronization in wireless sensor networks", IEEE Sensors Journal, Vol. 16, No. 13, pp. 5463-5470.

[4] Z. Wang, et al., (2017) "Cluster-based maximum consensus time synchronization for industrial wireless sensor networks", Sensors, Vol. 17, pp. 1-16, doi:10.3390/s17010141.

[5] J. He, et al., (2013) "Time synchronization in WSNs: A maximum-value-based consensus approach", IEEE Transactions on Automatic Control, Vol. 59, No. 3, pp. 660-675.

[6] Y. R. Faizulkhakov, (2007) "Time synchronization methods for wireless sensor networks: A survey", Programming and Computer Software, Vol. 33, No. 4, pp. 214-226.

[7] J. Li, et al., (2016) "Efficient time synchronization for structural health monitoring using wireless smart sensor networks", Structural Control and Health Monitoring, Vol. 23, No. 3, pp. 470-486.

[8] N. Sangjumpa, et al., (2016) "Analysis of adaptive multi-hop time synchronization in large wireless sensor networks", 7th International Conference of Information and Communication Technology for Embedded Systems (IC-ICTES), Bangkok, Thailand, pp. 79-84, doi:10.1109/ICTEmSys.2016.7467126.

[9] Z. G. Al-Mekhlafi, et al., (2019) "Firefly-inspired time synchronization mechanism for selforganizing energy-efficient wireless sensor networks: A survey", IEEE Access, Vol. 7, pp. 115229115248.

[10] S. Karthik, \& A. Ashok Kumar, (2020) "Ratings based energy-efficient clustering protocol for multihop routing in homogeneous sensor networks", International Journal of Intelligent Engineering and Systems, Vol. 13, No. 3, pp. 304-314.

[11] M. Xu, et al., (2014) "A Cluster-based secure synchronization protocol for underwater wireless sensor networks", International Journal of Distributed Sensor Networks, Vol 10, No. 4, pp. 1-13.

[12] M. Elsharief, et al., (2018) "FADS: Fast scheduling and accurate drift compensation for time synchronization of wireless sensor networks", IEEE Access, Vol. 6, pp. 65507-65520. 
International Journal of Computer Networks \& Communications (IJCNC) Vol.13, No.3, May 2021

[13] Y. P. Tian, et al., (2020) "Delay compensation-based time synchronization under random delays: Algorithm and Experiment", IEEE Transactions on Control Systems Technology, pp.1-16, doi:10.1109/TCST.2019.2956031.

[14] Z. Liu, et al., (2018) "Access control model based on time synchronization trust in wireless sensor networks", Sensors, Vol. 18, No. 7, pp. 1-15.

[15] J. He, et al., (2017) "Accurate clock synchronization in wireless sensor networks with bounded noise", Automatica, Vol. 81, pp. 350-358.

[16] Y. -P. Tian, et al., (2016) "Structural modeling and convergence analysis of consensus-based time synchronization algorithms over networks: Non-topological conditions”, Automatica, Vol. 65, pp. 64-75.

[17] X. Zhang et al., (2019) "RMTS: A robust clock synchronization scheme for wireless sensor networks", Journal of Network and Computer Applications, Vol. 135, pp. 1-10.

[18] X. Sun, X., et al., (2019) "Photovoltaic modules monitoring based on WSN with improved time synchronization", IEEE Access, Vol. 7, pp. 132406-132412.

[19] T. Qiu, et al., (2017) "SRTS: A self-recoverable time synchronization for sensor networks of healthcare IoT", Computer Networks, Vol. 129, pp. 481-492.

[20] D. Djenouri, et al., (2013) "Fast distributed multi-hop relative time synchronization protocol and estimators for wireless sensor networks", Ad Hoc Networks, Vol. 11, No. 8, pp. 2329-2344.

[21] S. Karthik, \& A. Ashok Kumar, (2015) "A constructive analysis of time synchronization in wireless sensor networks", International Journal of Computer Science and Technology, Vol. 6, No. 3, pp. 5660.

[22] J. Wang, et al., (2014) "Two-hop time synchronization protocol for sensor networks", EURASIP Journal on Wireless Communications and Networking, Vol. 39, pp. 1-10, doi:10.1186/1687-14992014-39.

[23] J. Wu, et al., (2015) "Cluster-based consensus time synchronization for wireless sensor networks", IEEE Sensors Journal, Vol. 15, No. 3, pp. 1404-1413.

[24] F. Shi, et al., (2019) "Novel maximum likelihood estimation of clock skew in one-way broadcast time synchronization”, IEEE Transactions on Industrial Electronics, Vol. 67, No. 11, pp. 9948-9957.

[25] H. Yan, et al., (2017) "Dynamic adjusting time synchronization for sensor networks", International Conference on Computer, Information and Telecommunication Systems (CITS), Dalian, China, doi:10.1109/CITS.2017.8035324.

[26] H. Wang, et al., (2017) "Estimation of frequency offset for time synchronization with immediate clock adjustment in multihop wireless sensor networks", IEEE Internet of Things Journal, Vol. 4, No. 6, pp. 2239-2246.

[27] F. Nunez, et al., (2017) "Pulse-coupled time synchronization for distributed acoustic event detection using wireless sensor networks", Control Engineering Practice, Vol. 60, pp. 106-117.

[28] N. Panigrahi, \& P.M. Khilar, (2017) "Multi-hop consensus time synchronization algorithm for sparse wireless sensor network: A distributed constraint-based dynamic programming approach", Ad Hoc Networks, Vol. 61, pp. 124-138.

[29] M. Muthumalathi, et al., (2019) "Efficient clock synchronization using energy based proportional integral and least common multiple protocol in wireless sensor networks", Journal of Engineering Science and Technology Review, Vol. 12, No. 4, pp. 144-151.

[30] G. S. S. Chalapathi, et al., (2019) "E-SATS: An efficient and simple time synchronization protocol for cluster-based wireless sensor networks", IEEE Sensors Journal, Vol. 19, No. 21, pp. $10144-$ 10156.

[31] D. Upadhyay, \& A. K. Dubey, (2020) "Maximum probable clock offset estimation (MPCOE) to reduce time synchronization problems in wireless sensor networks", Wireless Personal Communications, Vol. 114, No. 2, pp. 1177-1190.

[32] W. Heinzelman, et al., (2002) "An application-specific protocol architecture for wireless micro sensor networks", IEEE Transactions on Wireless Communications. Vol. 1, No. 4, pp. 660-670.

[33] S. Mirjalili, \& A. Lewis, (2016) "The Whale Optimization Algorithm", Advances in Engineering Software, Vol. 95, pp. 51-67. 
International Journal of Computer Networks \& Communications (IJCNC) Vol.13, No.3, May 2021

\section{AUTHORS}

Karthik Soundarapandian - received his Master of Science (I.T.) degree from Ramakrishna Mission Vivekananda College, University of Madras, Chennai (2009) and Master of Philosophy (C.S.) from Dr. G. R. Damodaran College of Science, Bharathiar University, Coimbatore (2011). Currently, he is working as Assistant Professor in Ramakrishna Mission Vivekananda College, and pursuing his Ph.D research in Bharathiar University, India. His research interest includes wireless communication, software engineering, data mining, and network security, etc.

Ashok Kumar Ambrose - received his Ph.D. from Manonmaniam Sundaranar University, Tirunelveli, India. Currently, he is working as Assistant Professor in the Department of Computer Science, Alagappa Government Arts College, Karaikudi, India. He has more than 20 years of experience in teaching. His research interest includes advanced networking, digital image processing, and computing algorithms.
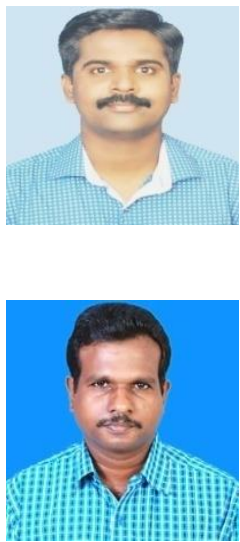